\title{
Valuation Bases for Extensions of Valued Vector Spaces *
}

\author{
Salma Kuhlmann
}

1. 6.1995

\begin{abstract}
Let $(V, v)$ be any valued vector space, and $\left(V_{0}, v\right)$ a subspace. Then $(V, v)$ admits a valuation basis over $\left(V_{0}, v\right)$ if and only if it admits a nice composition series over $\left(V_{0}, v\right)$. We show that this is always the case if $v\left(V \backslash V_{0}\right)$ is reversely well ordered. If $v\left(V_{0}\right)$ is reversely well ordered, we show that $V_{0}$ is nice in any extension, and that it admits a valuation basis over every subspace. Finally, we show that the property of admitting a valuation basis is preserved under countable dimensional extensions.
\end{abstract}

\section{Introduction}

Valued vector spaces played a historical role in the development of infinite abelian group theory in the last three decades. They were intensively used by group theorists seeking to generalize Ulm's structure theorem for countable $p$-groups (cf. [G] for a survey). Indeed, the socle of a p-group, endowed with the height function, may be viewed as a valued $I F_{p}$-vector space, with ordinal values (cf. [KAP] for these notions from abelian group theory, and [F1] for a comprehensive study on valued vector spaces and applications to $p$-groups and mixed groups). In this approach, the interest is often focused on that special case, where the valuation is the height function, and the value set an ordinal number. Our interest for valued vector spaces in the wide sense comes from a different direction; indeed they emerge quite naturally in the context of ordered and valued fields. For instance, Brown's theorem stating that a valued vector space of countable dimension admits a valuation basis (cf. [B]) was used by the author to show a structure theorem for countable exponential fields (cf. $[\mathrm{KS}]$ and $[\mathrm{K}-\mathrm{K}]$ ). Also, in the context of valuation theory of fields, an intensive study of general valued modules is now given in [KF]. In the present paper, we concentrate on the notion of valuation independence, and on the question of existence of a valuation basis. Nice subspaces play naturally an important role.

*This paper represents some results from the author's doctoral thesis. 
In Section 2, we recall some definitions and results that we shall need in the subsequent sections.

We begin Section 3 with the definition of a nice composition series for a valued vector space $V$ over a subspace $V_{0}$, and show the existence of such to be equivalent to that of a valuation basis over $V_{0}$ (Theorem 14). Note that it has been shown (cf. [H$\mathrm{W}]$, Theorem 3.8) that for a valuated $p$-group, the existence of a nice composition series is equivalent to that of a pseudo $p$-basis.

We then investigate some cases where conditions on the value set imply the existence of such composition series. Recall that valued vector spaces which have a finite value set enjoy two important properties: they admit a valuation basis over every subspace, and they are nice in every extension. We show (Corollary 16) that the same holds with the assumption that the value set is inversely well ordered (instead of finite). If for the extension $V_{0} \subset V$, we have that $v\left(V \backslash V_{0}\right)$ is reversely well ordered, we can even show that $V$ will admit a nice composition series over $V_{0}$ (Corollary 15).

Next, we define the quotient valuation on a quotient by a nice subspace (note that niceness is necessary since we work with arbitrary totally ordered value sets, not necessarily having suprema). This permits us to relativize the previous results of the section, and thus study subextensions $V_{0} \subset V_{1} \subset V$. For instance, we show (Corollary 20) that if the value set of $V_{1} / V_{0}$ is reversely well ordered, then $V_{1}$ inherits from $V_{0}$ the property of being nice, or that of being maximally valued.

We close section 3 with the definition of "defectless extensions" and show (Theorem 22 ) that the extension $V_{0} \subset V$ is defectless if and only if $V_{0}$ is nice in $V$. This notion of "defectless" comes from valuation theory: given an extension of valued fields, we may consider the larger field as a general valued vector space (in the sense of [F3]) over the smaller one. The extension is said to be defectless if every finitely generated subspace admits a valuation basis. In general, Theorem 22 will not hold, but it does in our case where the valuation is trivial on the ground field.

The last section is devoted to proving that a countable dimensional extension of a space admitting a valuation basis, admits a valuation basis as well (Corollary 24). This generalizes a result of Fuchs ([F2], Lemma 7 and Theorem 8) where this was shown to hold if the value set is an ordinal number.

\section{Preliminaries on valuation independence and niceness}

In the sequel, let $V$ be a vector space over a field $K$, and $\Gamma$ a totally ordered set with last element $\infty$. A surjective map

$$
v: V \longrightarrow \Gamma
$$

is a valuation on $V$ (and $(V, v)$ is a valued vector space) if for all $x, y \in V$ and $k \in K$, the following holds:

(V1) $v(x)=\infty$ if and only if $x=0$,

(V2) $v(k x)=v(x)$ if $k \neq 0$, 
(V3) $v(x+y) \geq \min \{v(x), v(y)\}$.

Condition (V3) is also called the triangle inequality. The following is a consequence of the above axioms:

$$
v(x) \neq v(y) \Longrightarrow v(x+y)=\min \{v(x), v(y)\} .
$$

The restriction of $v$ to a subspace $V_{0}$ of $V$ is a valuation on $V_{0}$ (that we denote also by $v)$. Now let $\left(V_{0}, v_{0}\right)$ and $(V, v)$ be valued vector spaces. Suppose that $V_{0}$ is a subspace of $V$ and that $v_{0}\left(V_{0}\right) \subset v(V)$. We will say that $(V, v)$ is an extension of $\left(V_{0}, v_{0}\right)$, and write $\left(V_{0}, v_{0}\right) \subset(V, v)$ if $v(x)=v_{0}(x)$ for all $x \in V_{0}$ (that is, $v_{0}$ is just the restriction of $v$ to $\left.V_{0}\right)$. We say that the extension $\left(V_{0}, v\right) \subset(V, v)$ is immediate if and only if for all nonzero $x \in V$ there exists $y \in V_{0}$ such that $v(x-y)>v(x)$. Note that if $\left(V_{0}, v\right) \subset(V, v)$ is a proper immediate extension, then $v\left(V \backslash V_{0}\right)$ contains an infinite increasing sequence. If $\left(V_{0}, v_{0}\right)$ admits no proper immediate extensions, we shall say that $V_{0}$ is maximally valued.

We say that $V_{0} \subset V$ is a finite extension (or $V$ is finitely generated over $V_{0}$ ) if and only if $V / V_{0}$ is of finite $K$-dimension.

If $\left\{x_{i} ; i \in I\right\} \subset V$, then

$$
\left\langle\left\{x_{i} ; i \in I\right\}\right\rangle
$$

will denote the $K$-subspace of $V$ generated by the elements $x_{i}$, that is the subspace consisting of all sums $\sum_{i \in I} k_{i} x_{i}$ where $k_{i}=0$ except for a finite number of $i \in I$ (by convention, $\langle\emptyset\rangle=0$ ). Let $\left\{V_{i} ; i \in I\right\}$ be a family of subspaces of $V$ such that $V=\bigoplus_{i \in I} V_{i}$ (i.e. $V$ is the direct sum of the $V_{i}^{\prime}$ s). We then say that $V$ is the coproduct of $\left\{V_{i} ; i \in I\right\}$ (or: $\left\{V_{i} ; i \in I\right\}$ is an orthogonal family of subspaces) if for every $\operatorname{sum} \sum_{i \in I} x_{i} \in V$ such that $x_{i} \in V_{i}$ for all $i \in I$ and $x_{i}=0$ except for a finite number of $i \in I$, then

$$
v\left(\sum_{i \in I} x_{i}\right)=\min _{i \in I} v\left(x_{i}\right) .
$$

We denote the coproduct of $\left\{V_{i} ; i \in I\right\}$ by $\perp_{i \in I} V_{i}$, and if $V_{i}$ is orthogonal to $V_{j}$, we write $V_{i} \perp V_{j}$.

Now let $V_{0}$ be a subspace of $V$ : The subset $\left\{x_{i} ; i \in I\right\} \subset V$ is independent over $V_{0}$ if and only if $\left\{x_{i}+V_{0} ; i \in I\right\}$ is $K$-linearly independent in $V / V_{0}$, and $\left\{x_{i} ; i \in I\right\}$ is a basis of $V$ over $V_{0}$ if and only if $\left\{x_{i}+V_{0} ; i \in I\right\}$ is a $K$-basis of $V / V_{0}$ (if and only if $\left.V=\bigoplus_{i \in I} K x_{i} \oplus V_{0}\right)$.

Note that if $\left\{x_{i} ; i \in I\right\}$ is independent over $V_{0}$, then in particular $\left\{x_{i} ; i \in I\right\}$ is $K$ - linearly independent.

We say that $\left\{x_{i} ; i \in I\right\} \subset V$ is valuation independent over $V_{0}$ if and only if $0 \notin\left\{x_{i} ; i \in I\right\}$, and for all $z_{0} \in V_{0}, k_{i} \in K$ such that $k_{i}=0$ except for a finite number of $i \in I$, we have

$$
v\left(\sum_{i \in I} k_{i} x_{i}+z_{0}\right)=\min _{\left\{i \in I ; k_{i} \neq 0\right\}}\left\{v\left(x_{i}\right), v\left(z_{0}\right)\right\} .
$$

If moreover $\left\{x_{i} ; i \in I\right\} \subset V$ is a $K$-basis of $V$ over $V_{0}$ then $\left\{x_{i} ; i \in I\right\}$ is a valuation basis of $V$ over $V_{0}$ (if and only if $V=\perp_{i \in I} K x_{i} \perp V_{0}$ ). 
Note that by definition, the element 0 never belongs to a valuation independent set. Also, by convention, $\min \emptyset=\infty$, and $\emptyset$ is valuation independent over $V_{0}$. The following lemma is easy to show:

Lemma 1 Let $\mathcal{B}, \mathcal{B}^{\prime}$ be two subsets of $V$. Then $\mathcal{B} \cup \mathcal{B}^{\prime}$ is valuation independent over $V_{0}$ if and only if $\mathcal{B}$ is valuation independent over $V_{0}$ and $\mathcal{B}^{\prime}$ is valuation independent over $\left\langle\mathcal{B}, V_{0}\right\rangle$. Moreover, the increasing union of a chain of valuation independent sets is again valuation independent.

Proposition 2 The subset $\left\{x_{i} ; i \in I\right\} \subset V \backslash\{0\}$ is valuation independent over $V_{0}$ if and only if for all $y \in\left\langle\left\{x_{i} ; i \in I\right\}\right\rangle$, if $y=\sum_{i \in I} k_{i} x_{i}$ then

$$
\max _{z \in V_{0}} v(y-z)=\min _{\left\{i \in I ; k_{i} \neq 0\right\}} v\left(x_{i}\right) \text {. }
$$

Proof: Clearly, $\left\{x_{i} ; i \in I\right\} \subset V$ is valuation independent over $V_{0}$ if and only if for every such sum $y \in\left\langle\left\{x_{i} ; i \in I\right\}\right\rangle$, and for all $z \in V_{0}$ we have

$$
v(y)=\min _{\left\{i \in I ; k_{i} \neq 0\right\}} v\left(x_{i}\right),
$$

and

$$
v(y+z)=\min \{v(y), v(z)\} .
$$

Now assume these last equations hold. Then certainly $v(y) \geq v(y+z)$, for all $z \in V_{0}$ whence $v(y)=\max _{z \in V_{0}} v(y-z)$. Conversely, if

$$
\max _{z \in V_{0}} v(y-z)=\min _{\left\{i \in I ; k_{i} \neq 0\right\}} v\left(x_{i}\right),
$$

then since on the other hand $v(y) \geq \min _{\left\{i \in I ; k_{i} \neq 0\right\}} v\left(x_{i}\right)$, equality holds. Moreover, we then have for all $z \in V_{0}$

$$
v(y) \geq v(y+z) \geq \min \{v(y), v(z)\}
$$

which shows that the last part of this inequality cannot be strict.

Remark 3 If $\left\{x_{i} ; i \in I\right\}$ is valuation independent over $V_{0}$ then $\left\{x_{i} ; i \in I\right\}$ is independent over $V_{0}$ : for if not we would have $k_{i}$, not all zero, $k_{i}=0$ except for a finite number of $i \in I$, such that $z_{0}=\sum_{i \in I} k_{i} x_{i} \in V_{0}$ and

$$
v\left(\sum_{i \in I} k_{i} x_{i}-z_{0}\right)=\infty \neq \min _{\left\{i \in I ; k_{i} \neq 0\right\}}\left\{v\left(x_{i}\right), v\left(z_{0}\right)\right\} .
$$

It follows that a valuation basis of $V$ over $V_{0}$ is always a maximal valuation independent set in $V$ over $V_{0}$.

Note that by Zorn's Lemma, there exists always in $V$ a (possibly empty) maximal valuation independent set $\mathcal{B}$ over $V_{0}$, and we have the following characterization of immediate extensions:

Lemma 4 Let $\mathcal{B}$ be maximal valuation independent in $V$ over $V_{0}$. Then $\mathcal{B}=\emptyset$ if and only if $V_{0} \subset V$ is an immediate extension. 
Proof: First note that by Proposition 2, if $0 \neq x \in V$ then $\{x\}$ is valuation independent over $V_{0}$ if and only if

$$
\max _{z \in V_{0}} v(x-z)=v(x)
$$

Now clearly, this last condition holds for some $x \in V$ if and only if the extension $V_{0} \subset V$ is not immediate, whence the result.

Theorem 5 For every $V_{0} \subset V$, there exists $V_{1}$ such that $V_{0} \subset V_{1} \subset V$ and

1) $V_{1}$ admits a valuation basis over $V_{0}$,

2) $V_{1} \subset V$ is an immediate extension.

Proof: $\quad$ Let $\mathcal{B} \subset V$ a maximal valuation independent set over $V_{0}$ and set

$$
V_{1}=\left\langle\mathcal{B} \cup V_{0}\right\rangle
$$

Obviously, $V_{1}$ satisfies 1 ). Moreover $\emptyset$ is maximal valuation independent in $V$ over $V_{1}$ : if not, there exists $\mathcal{B}^{\prime} \neq \emptyset, \mathcal{B}^{\prime} \subset V, \mathcal{B}^{\prime}$ valuation independent over $V_{1}$; it follows by Lemma 1 that $\mathcal{B} \cup \mathcal{B}^{\prime}$ is valuation independent over $V_{0}$, this contradicts the maximality of $\mathcal{B}$. Now by Lemma 4 , the extension $V_{1} \subset V$ is immediate.

Let us now recall the definition and basic properties of nice subspaces. We say that the subspace $V_{0}$ of $V$ is nice in $V$ if for all $x \in V$, the subset $v\left(x+V_{0}\right)=$ $\left\{v(y) ; y-x \in V_{0}\right\}$ of $v(V)$ admits a maximum. Now Let $x \in V$, and assume that the coset $x+V_{0}$ admits a representative $x_{0} \in x+V_{0}$ satisfying $v\left(x_{0}\right)=$ $\max v\left(x+V_{0}\right) \quad\left(x_{0}=0\right.$ if and only if $\left.x \in V_{0}\right)$. Such a representative is called a proper representative. Hence, $V_{0}$ is nice in $V$ if for all $x \in V$, the coset $x+V_{0}$ admits a proper representative.

Using Proposition 2, it is straightforward to show the following

Lemma 6 Let $x \in V \backslash V_{0}$ and $x_{0} \in x+V_{0}$. Then $x_{0}$ is a proper representative if and only if $\left\{x_{0}\right\}$ is a valuation basis of $\left\langle V_{0} \cup\{x\}\right\rangle$ over $V_{0}$. Consequently, $V_{0}$ is nice in $V$ if and only if for all $x \in V \backslash V_{0}$, the subspace $\left\langle V_{0} \cup\{x\}\right\rangle$ admits a valuation basis over $V_{0}$.

Note that if $V_{0}$ is nice in $V$, then $V_{0}$ is nice in every subextension as well. Also, $V_{0}$ is nice in $V$ if and only if for all $x \in V, V_{0}$ is nice in $\left\langle V_{0} \cup\{x\}\right\rangle$. Moreover, Lemma 4 together with the last lemma show that $V_{0}$ is nice in $\left\langle V_{0} \cup\{x\}\right\rangle$ if and only if the extension $V_{0} \subset\left\langle V_{0} \cup\{x\}\right\rangle$ is not immediate.

Corollary 7 Every maximal immediate extension $W$ of $V_{0}$ in $V$ is nice in $V$. In particular, if $V_{0}$ is maximally valued, then $V_{0}$ is nice in every extension $V$.

Proof: Let $V_{0} \subset W$ be a maximal immediate extension of $V_{0}$ in $V$. Suppose that $x \in V \backslash W$, then clearly the extension $W \subset\langle W \cup\{x\}\rangle$ cannot be immediate (otherwise, the extension $V_{0} \subset\langle W \cup\{x\}\rangle$ would be immediate as well, which contradicts the maximality of $W$ ). So by the note following Lemma $6, W$ is nice in $V$. 
Corollary 8 If $V$ admits a valuation basis over $V_{0}$, then $V_{0}$ is nice in $V$.

Proof: $\quad$ Let $\mathcal{B}$ the valuation basis of $V$ over $V_{0}$ and let $x \in V \backslash V_{0}$. Write $x=\sum_{i \in I} k_{i} b_{i}+z_{0}$ with $b_{i} \in \mathcal{B}$ and $z_{0} \in V_{0}$. Then by Proposition $2, v\left(x+V_{0}\right)$ admits $v\left(x-z_{0}\right)$ as its maximum. Hence $V_{0}$ is nice in $V$.

Note that the converse is in general not true.

Corollary 9 There exists $V_{1}$ such that $V_{0} \subset V_{1} \subset V$ and

1) $V_{0}$ is nice in $V_{1}$,

2) $V_{1} \subset V$ is an immediate extension.

Proof: Follows from Theorem 5 and Corollary 8.

In studying extensions $V_{0} \subset V$, with $V_{0}$ nice in $V$, it is often quite useful to consider the quotient space $V / V_{0}$. In this case, one can endow it with a canonical valuation $\bar{v}$ (see Lemma 10 below).

For the rest of this section, let us assume always that $V_{0}$ is nice in $V$.

Recall that then, every class $x+V_{0}$ has a proper representative. Define for all $x \in V$ :

$$
\bar{v}\left(x+V_{0}\right)=\max _{z \in V_{0}} v(x+z) ;
$$

this maximum exists for all $x \in V$. It is clear that $\bar{v}$ is well defined. Note that $\bar{v}\left(V / V_{0}\right) \subset v(V)$ and that $\bar{v}\left(x+V_{0}\right)=v(x)$ if and only if $x$ is a proper representative.

Lemma $10 \bar{v}$ is a valuation on $V / V_{0}$.

Proof: We have $\bar{v}\left(0+V_{0}\right)=\max _{z \in V_{0}} v(0+z)=\infty$, but if $x \notin V_{0}$, then $0 \notin x+V_{0}$ and $\bar{v}\left(x+V_{0}\right)=\max _{z \in V_{0}} v(x+z) \neq \infty$. Clearly, condition (V2) holds. Now we check the triangle inequality: Without loss of generality, let $x$ (respectively $y$ ) be a proper representative of $x+V_{0}$ (respectively of $y+V_{0}$ ). We then have

$$
\begin{aligned}
\bar{v}\left(x+y+V_{0}\right) & =\max _{z \in V_{0}} v(x+y+z) \geq v(x+y) \\
& \geq \min \{v(x), v(y)\}=\min \left\{\bar{v}\left(x+V_{0}\right), \bar{v}\left(y+V_{0}\right)\right\} .
\end{aligned}
$$

Note that in general, $x+y$ is not a proper representative of $x+y+V_{0}=\left(x+V_{0}\right)+$ $\left(y+V_{0}\right)$. But we have:

Lemma 11 Let $\left\{x_{i}+V_{0} ; i \in I\right\} \subset V / V_{0}$. Then $\left\{x_{i} ; i \in I\right\}$ is independent for the valuation $v$ over $V_{0}$ if and only if $\left\{x_{i}+V_{0} ; i \in I\right\}$ is independent for the valuation $\bar{v}$ and the $x_{i}$ are proper representatives. In this case, every finite sum $\sum_{i \in I} k_{i} x_{i}$ is a proper representative of $\sum_{i \in I} k_{i}\left(x_{i}+V_{0}\right)$. 
Proof: By Proposition 2, $\left\{x_{i} ; i \in I\right\} \subset V$ is independent for the valuation over $V_{0}$ if and only if for all $k_{i} \in K$ such that $k_{i}=0$ except for a finite number of $i \in I$,

$$
\max _{z \in V_{0}} v\left(\sum_{i \in I} k_{i} x_{i}+-z\right)=\min _{\left\{i \in I ; k_{i} \neq 0\right\}} v\left(x_{i}\right) \text {. }
$$

Take $k_{j}=0$ for $j \neq i$, this equation implies that $x_{i}$ is a proper representative. Since

$$
\max _{z \in V_{0}} v\left(\sum_{i \in I} k_{i} x_{i}-z\right)=\bar{v}\left(\sum_{i \in I} k_{i} x_{i}+V_{0}\right)=\bar{v}\left(\sum_{i \in I} k_{i}\left(x_{i}+V_{0}\right)\right)
$$

and

$$
\min _{\left\{i \in I ; k_{i} \neq 0\right\}} v\left(x_{i}\right)=\min _{\left\{i \in I ; k_{i} \neq 0\right\}} \bar{v}\left(x_{i}+V_{0}\right)
$$

for proper representatives $x_{i}$, the assertion of Proposition 2 is equivalent to the assertion of the lemma.

Lemma 12 Suppose $V_{0} \subset V_{1} \subset V$. Then $V_{1} \subset V$ is immediate if and only if $\left(V_{1} / V_{0}, \bar{v}\right) \subset\left(V / V_{0}, \bar{v}\right)$ is immediate.

Proof: $\quad \Rightarrow$ : Show that for all $x \in V$ there exists $y \in V_{1}$ such that $\bar{v}(x-$ $\left.y+V_{0}\right)>\bar{v}\left(x+V_{0}\right)$. Without loss of generality, $x$ is a proper representative, so $\bar{v}\left(x+V_{0}\right)=v(x)$. By hypothesis there exists $y \in V_{1}$ such that $v(x-y)>v(x)$, this implies

$$
\bar{v}\left(x-y+V_{0}\right)=\max _{z \in V_{0}} v(x-y+z) \geq v(x-y)>v(x)=\bar{v}\left(x+V_{0}\right) .
$$

$\Leftarrow$ : Suppose $\left(V_{1} / V_{0}, \bar{v}\right) \subset\left(V / V_{0}, \bar{v}\right)$ is immediate, and let $x \in V$. There exists $x_{1} \in V_{1}$ such that $\bar{v}\left(x-x_{1}+V_{0}\right)>\bar{v}\left(x+V_{0}\right)$, i.e.

$$
\max _{z \in V_{0}} v\left(x-x_{1}+z\right)>\max _{z \in V_{0}} v(x+z) \geq v(x) .
$$

Then there exists $z_{0} \in V_{0}$ such that $v\left(x-x_{1}+z_{0}\right)>v(x)$. Hence $y=x_{1}-z_{0} \in V_{1}$ satisfies $v(x-y)>v(x)$.

We close this section with the following well known result, which can be proved by standard arguments using the definition of niceness (see e.g. [KF], where this result is even proved for valued modules):

Corollary 13 Suppose that $V_{0} \subset V_{1} \subset V$. Then $V_{1}$ is nice in $V$ if and only if $V_{1} / V_{0}$ is nice in $V / V_{0}$.

\section{$3 \quad$ Nice subspaces and defectless extensions}

Let $V_{0}$ be a subspace of $V$. A nice composition series of $V$ over $V_{0}$ is a sequence $\left\{V_{\mu} ; 0 \leq \mu<\nu\right\}$ of subspaces, indexed by some ordinal $\nu$, such that

$V_{\mu}$ is nice in $V_{\mu+1}$ and $\operatorname{dim}\left(V_{\mu+1} / V_{\mu}\right) \leq 1$ if $\mu+1<\nu$,

$V_{\lambda}=\bigcup_{0 \leq \mu<\lambda} V_{\mu}$ for every limit ordinal $\lambda<\nu$ and $V=\bigcup_{0 \leq \mu<\nu} V_{\mu}$.

Obviously, if $V$ admits a nice composition series $\left\{V_{\mu} ; 0 \leq \mu<\nu\right\}$ over $V_{0}$, then it admits one over every $V_{\mu}$ as well. 
Theorem $14 V$ admits a nice composition series over $V_{0}$ if and only if $V$ admits a valuation basis over $V_{0}$.

Proof: Assume $V$ admits a nice composition series $\left\{V_{\mu} ; 0 \leq \mu<\nu\right\}$ over $V_{0}$. Hence, every $V_{\mu+1}$ admits a valuation basis $\left\{y_{\mu}\right\}$ over $V_{\mu}$. By induction and Lemma 1, $\left\{y_{\mu}, \ldots, y_{\mu+n}\right\}$ is a valuation basis of $V_{\mu+n+1}$ over $V_{\mu}$. Similarly, it follows from Lemma 1 that $\left\{y_{\mu} ; 0 \leq \mu<\lambda\right\}$ is a valuation basis of $V_{\lambda}$ over $V_{0}$ for every limit ordinal $\lambda<\nu$. By induction, we obtain the assertion.

Conversely, let $\mathcal{B}=\left\{y_{\mu} ; 0 \leq \mu<\nu\right\}$ be a valuation basis of $V$ over $V_{0}$. For $0 \leq \mu<\nu$, set

$$
V_{\mu}=\left\langle V_{0},\left\{y_{\alpha} ; \alpha<\mu\right\}\right\rangle .
$$

It follows again by Lemma 1 that $\left\{V_{\mu} ; 0 \leq \mu<\nu\right\}$ is a nice composition series of $V$ over $V_{0}$.

Let $\alpha$ be an ordinal. In the sequel, we shall denote by $\alpha^{*}$ the order type of the reversed ordering on $\alpha$.

Corollary 15 If $v\left(V \backslash V_{0}\right)=\alpha^{*}$, then $V$ admits a valuation basis over $V_{0}$ (in particular, $V_{0}$ is nice in $V$ ).

Proof: Without loss of generality, we may assume that $V$ is a proper extension of $V_{0}$. Let $\mathcal{B}=\left\{b_{\mu} ; 0 \leq \mu<\nu\right\}$ be a basis of $V$ over $V_{0}$. For $0 \leq \mu<\nu$, set

$$
V_{\mu}=\left\langle V_{0},\left\{b_{\alpha} ; \alpha<\mu\right\}\right\rangle \text {. }
$$

We show that $\left\{V_{\mu} ; 0 \leq \mu<\nu\right\}$ is a nice composition series of $V$ over $V_{0}$. We only have to show that $V_{\mu}$ is nice in $V_{\mu+1}$. But $v\left(V_{\mu+1} \backslash V_{\mu}\right) \subset v\left(V \backslash V_{0}\right)$. Thus $v\left(V_{\mu+1} \backslash V_{\mu}\right)$ is the reverse of an ordinal as well and hence contains no infinite increasing sequences. It follows that the extension $V_{\mu} \subset V_{\mu+1}$ is not immediate, hence the assertion.

Corollary 16 If $v\left(V_{0}\right)=\alpha^{*}$, then $V_{0}$ is nice in $V$. Further, $V_{0}$ admits a valuation basis over every subspace.

Proof: The hypothesis implies that for all $x \in V, v\left(V_{0}+K x\right)$ is also the reverse of an ordinal, and so is $v\left(V_{0}+K x \backslash V_{0}\right)$. Then by Corollary $15, V_{0}$ is nice in $V_{0}+K x$. Now let $W$ be a subspace of $V_{0}$. Then $v\left(V_{0} \backslash W\right) \subset v\left(V_{0}\right)$, hence $v\left(V_{0} \backslash W\right)$ is also the reverse of an ordinal. The second assertion follows now by Corollary 15.

Note that in the last corollary, $V$ will not necessarily admit a valuation basis over $V_{0}$.

Corollary 17 If $\operatorname{dim} V \leq \aleph_{0}$, then $V$ admits a valuation basis over every subspace $V_{0}$ of finite dimension. 
Proof: Write $V=\bigcup_{1<\mu<\aleph_{0}} V_{\mu}$ where the $V_{\mu}$ are subspaces of finite dimension and $\operatorname{dim} V_{\mu+1} / V_{\mu} \leq 1$. The dimension of $V_{\mu}$ being finite, $v\left(V_{\mu}\right)$ is finite; then by Corollary $16, V_{\mu}$ is nice in $V_{\mu+1}$. Now, the assertion follows from Theorem 14 .

We have seen in Theorem 14 that a space $V$ admits a valuation basis over a given nice subspace $V_{0}$ if and only if $V$ has a nice composition series over $V_{0}$. But of course, such a composition series does not always exist. For instance, the 0 space is nice in every space $V$, but not every $V$ admits a valuation basis. However, we shall now describe some more cases where niceness implies the existence of nice composition series. To this end, we shall apply the results so far obtained in this section to the valued vector space $\left(V / V_{0}, \bar{v}\right)$, and this in turn will give us information about the extension $V_{0} \subset V$.

Proposition 18 Let $V_{0}$ be nice in $V$. Then $\left(V / V_{0}, \bar{v}\right)$ admits a valuation basis if and only if $V$ admits a valuation basis over $V_{0}$.

Proof: Follows by Lemma 11.

From this proposition and Corollary 17, we obtain the following strengthening of Corollary 17.

Corollary 19 Let $V_{0}$ be nice in $V$. If $\operatorname{dim} V / V_{0} \leq \aleph_{0}$, then $V$ admits a valuation basis over $V_{0}$.

Note that this last corollary gives the converse to Corollary 8 in the case of countable dimensional extensions.

Corollary 20 Let $V_{0}$ be nice in $V$. Let $V_{0} \subset V_{1} \subset V$ and $\bar{v}\left(V_{1} / V_{0}\right)=\alpha^{*}$. Then $V_{1}$ admits a valuation basis over $V_{0}$ and $V_{1}$ is again nice in $V$. If moreover $V_{0}$ is maximally valued, then $V_{1}$ is maximally valued as well.

Proof: Since $\bar{v}\left(V_{1} / V_{0}\right)=\alpha^{*}$, then $V_{1} / V_{0}$ admits a valuation basis over $\{0\}$ (by Corollary 16). Hence by Proposition $18, V_{1}$ admits a valuation basis over $V_{0}$. By Corollary $16, V_{1} / V_{0}$ is nice in $V / V_{0}$, and by Corollary 13 it follows that $V_{1}$ is nice in $V$.

If moreover $V_{0}$ is maximally valued, then $V_{0}$ is nice in every extension $V^{\prime}$ of $V_{1}$ and hence $V_{1}$ is nice in every extension $V^{\prime}$. This implies by the note following Lemma 6 that $V_{1}$ admits no proper immediate extension.

As a corollary, we get yet another generalization of Corollary 17:

Corollary 21 Suppose that $V$ admits a valuation basis over $V_{0}$, and let $V_{0} \subset V_{1} \subset V$ such that $V_{0} \subset V_{1}$ is a finite extension. Then $V$ admits a valuation basis over $V_{1}$. 
Proof: $\quad$ Let $\mathcal{B}$ a valuation basis over $V_{0}$. Since $V_{0} \subset V_{1}$ is finite, there exists a partition $\mathcal{B}=\mathcal{B}^{\prime} \cdot \mathcal{B}^{\prime \prime}$ such that $\mathcal{B}^{\prime}$ is finite and $V_{1} \subset\left\langle\mathcal{B}^{\prime}, V_{0}\right\rangle=: W$. By Corollary 8 , $V_{0}$ is nice in $V$. So by Corollary $20, V_{1}$ is nice in $V$ and hence in $W$. Then by Corollary $19, W$ admits a valuation basis $\mathcal{B}^{*}$ over $V_{1}$. By Lemma $1, \mathcal{B}^{\prime \prime}$ is a valuation basis of $V$ over $W$ and $\mathcal{B}^{\prime \prime} \cup \mathcal{B}^{*}$ is a valuation basis of $V$ over $V_{1}$.

We say that $V_{0} \subset V$ is a defectless extension if and only if for every finite subextension $V_{0} \subset V_{1}$, there exists a valuation basis of $V_{1}$ over $V_{0}$. Note that if $V_{0} \subset W \subset V$ and if $V_{0} \subset V$ is a defectless extension, then $V_{0} \subset W$ is a defectless extension.

Theorem $22 V_{0}$ is nice in $V$ if and only if $V_{0} \subset V$ is a defectless extension.

Proof: $\Leftarrow$ : follows from the definition and Lemma 2.6.

$\Rightarrow$ : If $V_{0}$ is nice in $V$, then by Corollary 20, every finite extension of $V_{0}$ admits a valuation basis over $V_{0}$, hence $V_{0} \subset V$ is a defectless extension.

\section{Extensions of spaces admitting a valuation ba- sis}

Theorem 23 Suppose that $V_{0}$ admits a valuation basis and that $V_{0} \subset V$ is immediate. Let $W \subset V$ such that

$$
V=V_{0} \oplus W
$$

then there exist $V_{1}, V_{2} \subset V_{0}$ such that

i) $V_{2} \subset V_{2} \oplus W$ is immediate and $\operatorname{dim} V_{2} \leq \max \left\{\aleph_{0}, \operatorname{dim} W\right\}$

ii) $V_{1}$ and $V_{2}$ admit a valuation basis

iii) $V_{0}=V_{1} \perp V_{2}$, and hence

$$
V=V_{1} \perp\left(V_{2} \oplus W\right)
$$

Proof: $\quad$ Let $\mathcal{B}$ a valuation basis of $V_{0}$. We first show the following fact: if $U$ is a subspace of $V$, then there exists $\mathcal{B}_{U} \subset \mathcal{B}$ such that

$$
\operatorname{card} \mathcal{B}_{U} \leq \max \left\{\aleph_{0}, \operatorname{dim} U\right\}
$$

and

$$
\forall 0 \neq u \in U \exists y \in\left\langle\mathcal{B}_{U}\right\rangle: v(u-y)>v(u) .
$$

For let $\left\{u_{i} ; i \in I\right\}$ be a maximal valuation independent set in $U$. Then the extension $\left\langle u_{i} ; i \in I\right\rangle \subset U$ is immediate (cf. Lemma 4), and card $\left\{u_{i} ; i \in I\right\} \leq \operatorname{dim} U$ (cf. Remark 3). Further, since $V_{0} \subset V$ is immediate, for all $i \in I$ there exists a finite subset $\mathcal{B}_{i} \subset \mathcal{B}$ and $y_{i} \in\left\langle\mathcal{B}_{i}\right\rangle$ such that

$$
v\left(u_{i}-y_{i}\right)>v\left(u_{i}\right) .
$$


Set $\mathcal{B}_{U}=\bigcup_{i \in I} \mathcal{B}_{i}$, then if $\sum k_{i} u_{i} \in\left\langle u_{i} ; i \in I\right\rangle$, we have $\sum k_{i} y_{i} \in\left\langle\mathcal{B}_{U}\right\rangle$ and

$$
\begin{aligned}
v\left(\sum k_{i} u_{i}-\sum k_{i} y_{i}\right) & =v\left(\sum k_{i}\left(u_{i}-y_{i}\right)\right) \geq \min _{i \in I ; k_{i} \neq 0} v\left(u_{i}-y_{i}\right) \\
& >\min _{i \in I ; k_{i} \neq 0} v\left(u_{i}\right)=v\left(\sum k_{i} u_{i}\right),
\end{aligned}
$$

hence $\mathcal{B}_{U}$ satisfies assertion (1) for $0 \neq u \in\left\langle u_{i} ; i \in I\right\rangle$. We claim now that $\mathcal{B}_{U}$ satisfies assertion (1) for all $0 \neq u \in U$. Indeed let $0 \neq u \in U$. Since the extension $\left\langle u_{i} ; i \in I\right\rangle \subset U$ is immediate, let $u^{\prime} \in\left\langle u_{i} ; i \in I\right\rangle$ such that $v\left(u-u^{\prime}\right)>v(u)$. Now let $y \in\left\langle\mathcal{B}_{U}\right\rangle$ such that $v\left(u^{\prime}-y\right)>v\left(u^{\prime}\right)$. A straightforward argument, using the triangle inequality, shows then that $v(u-y)>v(u)$.

Let us define by induction on $n \in \omega$ an increasing sequence $\mathcal{B}_{n}$ of subsets of $\mathcal{B}$ as follows:

$$
\mathcal{B}_{1}=\mathcal{B}_{W} \text { and } \mathcal{B}_{n+1}=\mathcal{B}_{n} \cup \mathcal{B}_{\left\langle\mathcal{B}_{n}, W\right\rangle}
$$

Set

$$
\mathcal{B}_{\omega}=\bigcup_{n \in \omega} \mathcal{B}_{n} \text { and } V_{2}=\left\langle\mathcal{B}_{\omega}\right\rangle
$$

Hence $\operatorname{dim} V_{2}=\operatorname{card} \mathcal{B}_{\omega} \leq \max \left\{\aleph_{0}, \operatorname{dim} W\right\}$. If $0 \neq x \in V_{2} \oplus W$, then there exists $n \in \omega$ such that $x \in\left\langle\mathcal{B}_{n}, W\right\rangle$. Consequently, there exists $y \in\left\langle\mathcal{B}_{n+1}\right\rangle$ such that

$$
v(x-y)>v(x),
$$

which implies assertion i).

Set $V_{1}=\left\langle\mathcal{B} \backslash \mathcal{B}_{\omega}\right\rangle$. Assertion ii) is clear, and also that $V_{0}=V_{1} \perp V_{2}$. To prove assertion iii) that $V=V_{1} \perp V_{2} \oplus W$, it suffices to verify the following general fact: if $V_{i} \perp V_{j}$ and $V_{j} \subset V_{j^{\prime}}$ is immediate, then $V_{i} \perp V_{j^{\prime}}$. The proof is straightforward.

Corollary 24 Suppose that $V_{0}$ admits a valuation basis and that $\operatorname{dim}\left(V / V_{0}\right) \leq \aleph_{0}$. Then $V$ admits a valuation basis.

Proof: By Theorem 5 and Lemma 1, it is clear that it suffices to consider the case where $V_{0} \subset V$ is immediate. By hypothesis, there exists $W \subset V$ with $\operatorname{dim} W \leq \aleph_{0}$ and

$$
V=V_{0} \oplus W
$$

Let

$$
V=V_{1} \perp\left(V_{2} \oplus W\right)
$$

be the corresponding decomposition of $V$, as described in Theorem 23 above. Since $\operatorname{dim}\left(V_{2} \oplus W\right) \leq \aleph_{0}$, it follows by Corollary 17 that $V_{2} \oplus W$ admits a valuation basis. To see that $V$ admits a valuation basis, it suffices to verify the following general fact: if $V_{i}$ admits a valuation basis $\mathcal{B}_{i}$ for all $i \in I$, then $V=\perp_{i \in I} V_{i}$ admits $\bigcup_{i \in I} \mathcal{B}_{i}$ as a valuation basis. The proof is straightforward. 


\section{References}

[B] Brown, R.: Valued vector spaces of countable dimension, Publ. Math. Debrecen 18 (1971), 149-151

[F1] Fuchs, L.: Abelian $p$-groups and mixed groups, Montréal (1980)

[F2] Fuchs, L.: Subfree valued vector spaces, Abelian group theory, Proc. 2nd New Mex. State Univ. Conf. 1976, Springer LNM 616, (1977)

[F3] Fuchs, L.: Vector spaces with general valuations, Gruppi e Anelli Ordin., Convegno 1975, Symp. Math. 21 (1977), 433-450

[G] Göbel, R.: Helmut Ulm: His Work and His Impact on Recent Mathematics, in: Contemporary Mathematics 87 (1989)

[H-W] Hunter, R. and Walker, E.: Valuated p-groups, Abelian group theory, Proc. Oberwolfach 1981, eds. R. Göbel \& E. Walker, Springer LNM 874 (1981)

[KAP] Kaplansky, I.: Infinite Abelian Groups, Ann Arbor (1954)

[KF] Kuhlmann, F.-V.: Valuation Theory of Fields, Abelian Groups and Modules, to appear in the "Algebra, Logic and Applications" series, eds. Göbel and Macintyre, by Gordon and Breach.

[K-K] Kuhlmann, F.-V.- Kuhlmann, S. : On the structure of nonarchimedean exponential fields II, Comm. in Algebra 22(12) (1994), 5079-5103

[KS] Kuhlmann, S.: On the structure of nonarchimedean exponential fields I, to appear in Arch. Math. Logic

Mathematisches Institut

Universität Heidelberg

Im Neuenheimer Feld 288

D-69120 Heidelberg, Germany 